(1)

CrossMark

\title{
An evaluation of a throat discomfort visual analogue scale in chronic cough
}

\author{
To the Editor:
}

Chronic cough is a debilitating symptom affecting approximately one in ten of the general population and leading to impaired quality of life and social exclusion [1]. Many individuals with chronic cough localise their symptoms to the throat area and report sensations such as a "persistent tickling", "irritation" or "blockage" in the throat [2]. They also often describe that symptoms are provoked by non-specific environmental triggers, such as exposure to scents or odours. In many patients, there is also an apparent overlap between manifestations of laryngeal dysfunction, such as discomfort on swallowing with or without changes in voice quality. Overall, these phenomena may be attributed to an underlying laryngeal hypersensitivity (LHS) [3] and recognition of this entity in chronic cough may be important to help inform and select the optimum treatment strategy; e.g. preferential specialist speech and language therapy (SLT) intervention [4].

Currently, the most widely utilised questionnaire for identifying LHS is the Newcastle Laryngeal Hypersensitivity Questionnaire (LHQ) [5]. VerTigan et al. [5] demonstrated that this 14-item questionnaire, which focusses on abnormal laryngeal sensation across three domains of obstruction, pain/ thermal and irritation, could identify patients with laryngeal-focused symptomatology and also potentially detect favourable response to SLT therapy.

In practice, the completion and scoring of this questionnaire is time-intensive, thereby somewhat limiting its practical application in everyday clinical practice. A questionnaire-based assessment approach in chronic cough has faced the same challenge and thus many clinical centres and indeed research study protocols have increasingly defaulted to utilising a simple $10-\mathrm{cm}$ visual analogue scale (VAS) to characterise and monitor chronic cough during treatment $[6,7]$. For some time, we have been utilising a throat/laryngeal-focused VAS in clinical practice and the aim of this brief communication is to describe the efficacy and validity of this approach, in providing a simple means of capturing laryngeal symptoms with or without hypersensitivity.

All patients referred for SLT from the Royal Brompton Hospital (London, UK) upper airway clinic between 2017 and 2019 were invited, at initial attendance, as part of our standard clinical assessment protocol, to complete a throat discomfort VAS (anchor terms: 0: no discomfort at all; 10: worst discomfort ever), Leicester Cough Questionnaire (LCQ) [8] and LHQ (with a score $\leqslant 17$ taken as abnormal). These measures were re-assessed in a sub-group of patients following SLT treatment. The project was registered with the Royal Brompton and Harefield NHS Foundation Trust service evaluation team (CIRIS project ID 2787) with all data anonymised and analysed as part of our standard care.

88 patients (median age 56 years, range 17-82 years; 65\% female) completed all three rating tools. 24 patients had a co-existing diagnosis of asthma. Mean \pm SD throat discomfort VAS score was $3.9 \pm 2.9$, with a significantly higher mean score for males $(5.0 \pm 2.9)$ than females $(3.4 \pm 2.8)(p=0.01)$. There was no correlation between throat VAS and age $(r=-0.02, \mathrm{p}=0.86)$.

There was an inverse relationship between throat VAS and LHQ $(r=-0.4, \mathrm{p}<0.01)$ but no relationship between throat VAS and LCQ $(\mathrm{p}>0.05)$. ROC analysis (figure 1a) revealed a cut-off throat VAS $>3$ yielded a sensitivity of 0.59 and a specificity of 0.77 to detect LHQ $\leqslant 17$. Yet, out of 47 patients with an LHQ score of $\leqslant 17,44$ had a throat VAS of $>3$ corresponding to a positive predictive value in this cohort of $94 \%$. The negative predictive value (NPV) was however only $24 \%$ as 10 out of 41 had an LHQ score of $>17$ and a

@ERSpublications

Chronic cough (CC) is common. Recognition of laryngeal hypersensitivity (LHS) allows for targeted treatment. A throat visual analogue scale (VAS) allows for a rapid and simple method to detect LHS in CC and identifying targeted intervention. http://bit.ly/32zPFiK

Cite this article as: Hull JH, Walsted ES, Pavitt MJ, et al. An evaluation of a throat discomfort visual analogue scale in chronic cough. Eur Respir J 2020; 55: 1901722 [https://doi.org/10.1183/13993003.017222019]. 

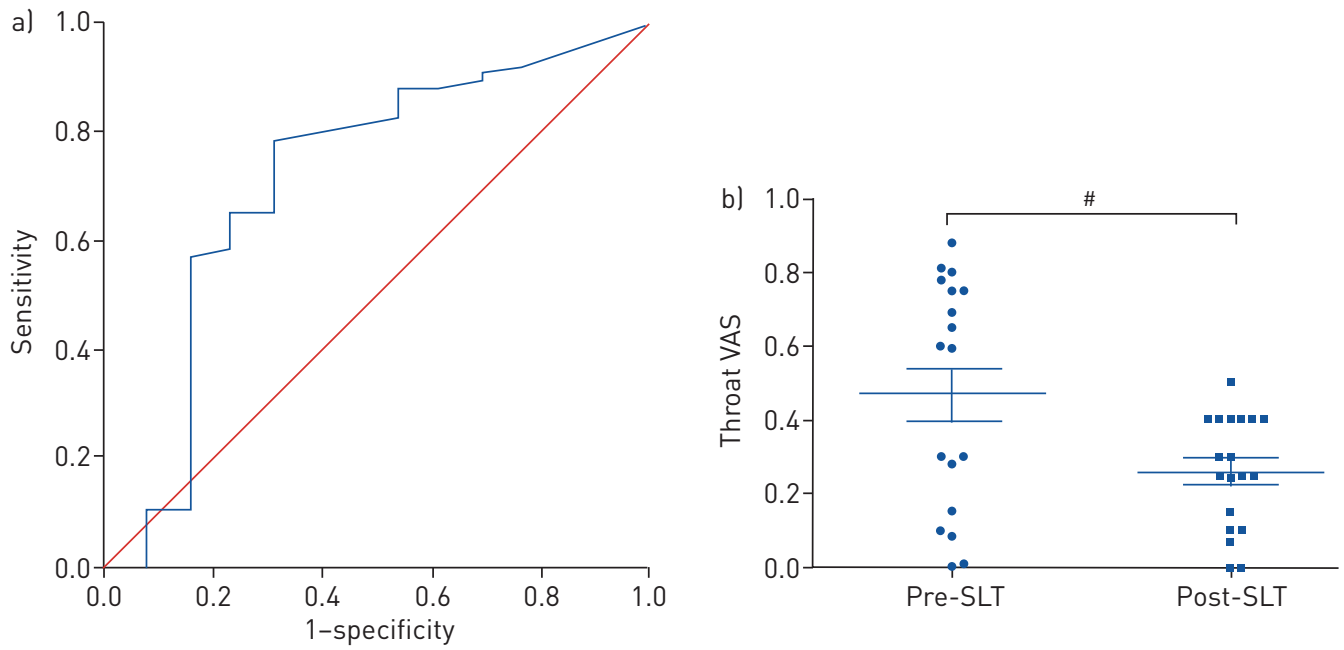

FIGURE 1 a) Receiver operator characteristics (ROC) of a throat visual analogue scale (VAS) to detect laryngeal hypersensitivity as identified by Newcastle Laryngeal Hypersensitivity Questionnaire score $\leqslant 17$. b) Change in throat VAS scores pre- and post-speech and language therapy (SLT) intervention. Data presented as individual data points with the median and interquartile range displayed as solid lines. Wilcoxon-sign rank test was used to compare change in throat VAS pre- and post-SLT intervention, median change $-2.3(-5.3-1.2)$; \#: $\mathrm{p}=0.02$.

throat VAS of $\leqslant 3$. Pre- to post-treatment ratings $(n=19$, median age $=52$ years, range $16-75$ years; $83 \%$ female) showed a reduction in throat VAS (median (IQR)) -2.3 (-5.3, 1.2) $(\mathrm{p}=0.02)$ after SLT intervention (figure $1 \mathrm{~b}$ ).

In the subgroup of patients who received SLT $(n=19)$, the decrease in throat VAS from pre- to post-intervention was closely correlated with the pre-intervention throat VAS $\left(\mathrm{r}^{2}=0.9 ; \mathrm{p}<0.01\right)$. Acknowledging a possible effect from regression towards the mean, a decrease was seen in 15 (79\%) out of 19 patients, with a mean \pm SD decrease of $82 \pm 16 \%$ whilst the four non-responders had a pre-intervention throat VAS of $\leqslant 1$.

A direct relationship exists between the throat VAS and LHQ, indicating that a VAS-based approach may provide a simple and rapid means for detecting LHS in patients with chronic cough. In this clinically pragmatic and thus preliminary evaluation, a VAS score of $>3$ appeared to positively identify patients with throat discomfort and LHS, with a response to SLT-based intervention. A VAS-based approach may thus obviate the need for a lengthy and more detailed rating of the specific nature of discomfort. The specificity of 0.77 suggests that use of a VAS cut-off at 3.0 is indicative of LHS, with a low false positive rate, i.e. a throat VAS score of $>3$ identifies individuals likely to have a low LHQ but if less than 3 , does not rule out the presence of LHS.

A throat discomfort VAS offers the potential to assess and characterise the severity of a patient's symptoms, and to identify patients likely to respond to SLT. In the current study we used a scale that did not utilise anchor terms throughout the scale (e.g. mild, moderate, severe). The VAS may thus benefit from anchor terms to aid future application and interpretation but also to facilitate future work to establish the minimally clinical important difference; i.e. to assess meaningful change. Equally, it may be that terms other than "discomfort" could be employed (e.g. itch). In the current work we employed this term following engagement with patient expert groups.

The detection of LHS is likely to be important in helping identify those individuals who may benefit most from SLT intervention. This is also likely to become important for the assessment of any novel treatments targeting laryngeal desensitisation. It also remains important to assess and treat other well recognised triggers for LHS including nasal disease and gastro-oesophageal reflux. Overall, this work therefore signals the potential of a tool with the potential to preferentially select individuals who may benefit from upper airway intervention. Moreover, the improvement from pre- to post-treatment ratings indicates that a VAS-based approach may also be relevant in assessing changes in laryngeal symptoms over time.

A key limitation in this field is the current lack of a "gold-standard" means for assessing LHS [9]. Several methods have been proposed including electromyography-based techniques but these are dependent on assessment of laryngeal motor output. Moreover, the historical use of sensory testing methodology (e.g. Fiberoptic Endoscopic Evaluation of Swallowing with Sensory Testing) has fallen out of favour 
because of difficulties with the reliability of endoscopic placement. Future techniques and methodologies should focus on providing a more robust means of assessing laryngeal sensory function and indeed these techniques need to assess function in the context of provocation or the presence of some form of trigger, to be truly valid [10].

This work now requires validation in a prospective multi-centre study in a more generalisable patient population, to determine the true validity of a VAS-based approach to the detection of LHS in individuals with laryngeal-focused cough and other respiratory symptoms.

James H. Hull $\oplus^{1}$, Emil S. Walsted $\oplus^{1,2}$, Matthew J. Pavitt $\odot^{1}$, Bethany Tidmarsh ${ }^{1}$ and Julia Selby ${ }^{1}$

${ }^{1}$ Dept of Respiratory Medicine, Royal Brompton Hospital, London, UK. ${ }^{2}$ Dept of Respiratory Medicine, Bispebjerg Hospital, Copenhagen, Denmark.

Correspondence: James H. Hull, Dept of Respiratory Medicine, Royal Brompton Hospital, London, SW3 6HP, UK. E-mail: j.hull@rbht.nhs.uk

Received: 3 Sept 2019 | Accepted after revision: 31 Oct 2019

Individual participant data that underlie the results in the article, after de-identification (text, tables, figures and appendices) will be available on request. Data will be available to anyone who wishes to access the data, for any purpose.

Author contributions: J.H. Hull and J. Selby developed the idea behind the study. B. Tidmarsh and J. Selby undertook patient visits and data collection. M.J. Pavitt and E.S. Walsted undertook the data analysis. J.H. Hull wrote the first draft of the manuscript. All authors contributed to the final manuscript. J.H. Hull and J. Selby confirm full responsibility for the content of the manuscript. J.H. Hull affirms that this manuscript is an honest, accurate, and transparent account of the data being reported; that no important aspects of the study have been omitted.

Conflict of interest: None declared.

\section{References}

1 Morice AH, Jakes AD, Faruqi S, et al. A worldwide survey of chronic cough: a manifestation of enhanced somatosensory response. Eur Respir J 2014; 44: 1149-1155.

2 Vertigan AE, Gibson PG. Chronic refractory cough as a sensory neuropathy: evidence from a reinterpretation of cough triggers. J Voice 2011; 25: 596-601.

3 Hull JH, Menon A. Laryngeal hypersensitivity in chronic cough. Pulm Pharmacol Ther 2015; 35: 111-116.

4 Chamberlain Mitchell SA, Garrod R, Clark L, et al. Physiotherapy, and speech and language therapy intervention for patients with refractory chronic cough: a multicentre randomised control trial. Thorax 2017; 72: 129-136.

5 Vertigan AE, Bone SL, Gibson PG. Development and validation of the Newcastle laryngeal hypersensitivity questionnaire. Cough 2014; 10: 1.

6 Morice AH, Millqvist E, Bieksiene K, et al. ERS guidelines on the diagnosis and treatment of chronic cough in adults and children. Eur Respir J 2020; 55: 1901136.

7 Spinou A, Birring SS. An update on measurement and monitoring of cough: what are the important study endpoints? J Thorac Dis 2014; 6: S728-S734.

8 Birring SS, Prudon B, Carr AJ, et al. Development of a symptom specific health status measure for patients with chronic cough: Leicester Cough Questionnaire (LCQ). Thorax 2003; 58: 339-343.

9 Famokunwa B, Walsted ES, Hull JH. Assessing laryngeal function and hypersensitivity. Pulm Pharmacol Ther 2019; 56: 108-115.

10 Hull JH, Walsted ES, Feary J, et al. Continuous laryngoscopy during provocation in the assessment of inducible laryngeal obstruction. Laryngoscope 2019; 129: 1863-1866. 